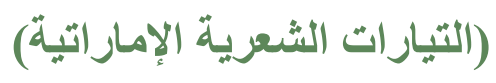

\title{
Emirati Poetic Movements
}

\author{
Zainab ALYASI ${ }^{1}$ (1)
}

'United Emirates University, Department of Arabic Language and Literature, Dubai

\section{ORCID: Z.A. 0000-0003-1771-0722}

Sorumlu yazar/Corresponding author: Zainab ALYASI (Dr.),

United Emirates University, Department of Arabic Language and Literature, Dubai

E-posta:zalyase14@hotmail.com

Başvuru/Submitted: 03.10.2020 Revizyon Talebi/Revision Requested: 19.10.2020

Son Revizyon/Last Revision Received: 11.11.2020

Kabul/Accepted: 11.12 .2020

Atıf/Citation: Alyasi, Zainab. "Emirati Poetic Movements". Şarkiyat Mecmuası - Journal of Oriental Studies 37 (2020), 109-125.

https://doi.org/10.26650/jos.2020.009

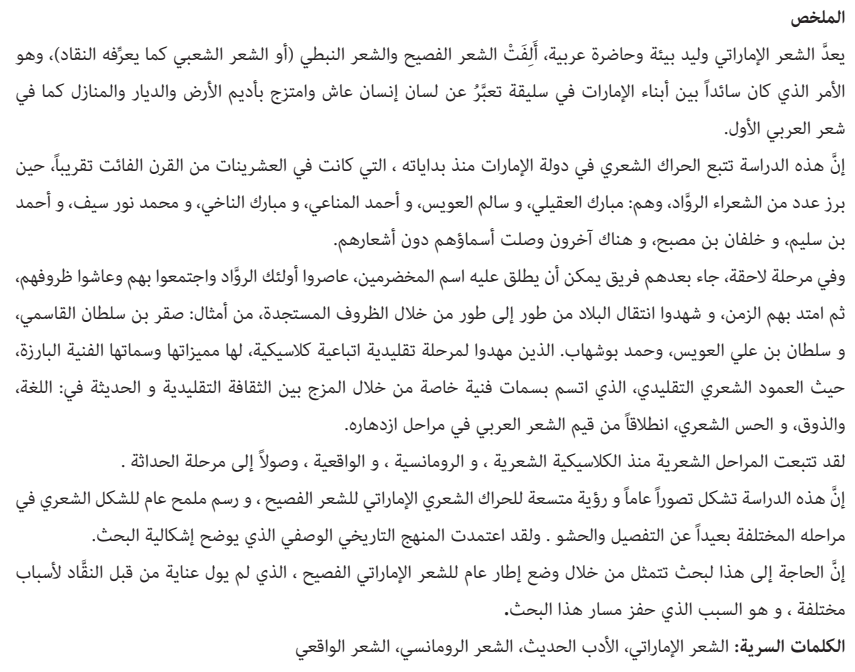

الملخص

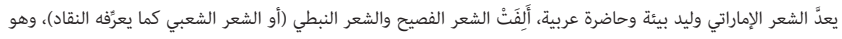

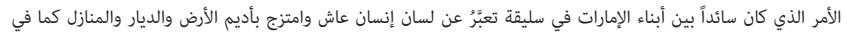
شعر العربي الأول. - مان.

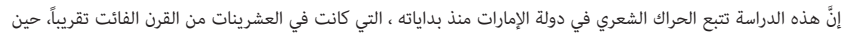

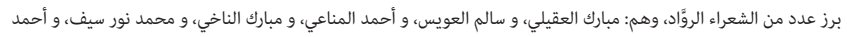
بن سليم، و خلفان بن مصبح، و هناك آخرون وصلت أسماؤهم دون أشعاد أشعارهم.

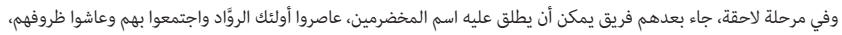

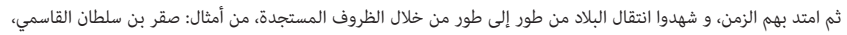

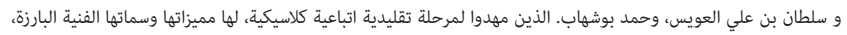

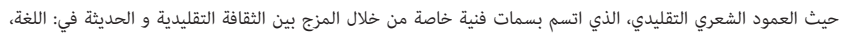

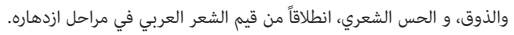

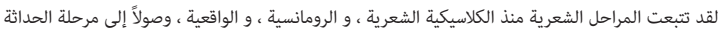

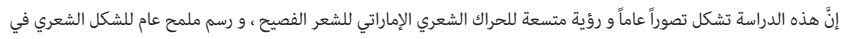

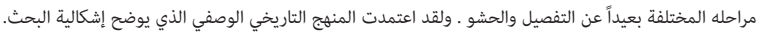

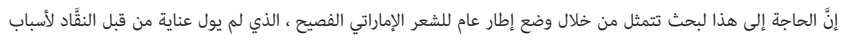

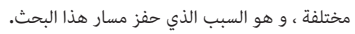
الكلمات السرية: الشعر الإماراتي، الأدب الحديث، الشعر الرومانسي، الشعر الواقعي

\section{ABSTRACT}

Emirati poetry is considered the fruit of an Arabic environment that was drawn to formal Arabic poetry and colloquial poetry (or "folk poetry" as defined by critics). That was what was common within the Emirati people. The poets had an innate ability to express the thoughts of a person who lived and integrated with the land and peoples' homes as found in the original Arabic poetry. The beginning of Emirates poetry was around the 1920s. During that time, several pioneer poets shined, including Mubarak Al Aqili, Salem Al Owais, Ahmed Al Mannai, Mubarak Al Nakhi, Muhammad Nour Saif, Ahmed Bin Salem, Khalfan Bin Musbah, and others, whose names survive, although their poetry did not. After the original poets, another group that came to be known as veterans. They followed the early pioneers, met them, and lived as they did. However, their influence and popularity sustained for longer. They witnessed the many political and sociological phases that the country went through. These veterans include Saqr bin Sultan AI Qasimi, Sultan Bin Ali Al Owais, and Hamad Bu shehab. They paved the way for a classical subordinate traditional phase, with its distinctive artistic features and characteristics. It included traditional column poetry with its unique artistic features that mixed traditional and modern culture through 
language, taste, and poetic sense. It retained a base in the values of Arabic poetry in its early stages. The Classical phase was followed by a Romantic stage, led by the poet Manee Saeed Al Otaiba. A period of Realism came after, which kept pace with Arab reality and its circumstances. One of the primary poets of this stage was Muhammad Sharif Al-Shaibani.

Keywords: Emirati Poetry, modern literature, romantic poem, realistic poem

\section{EXTENDED ABSTRACT}

Discussing a nation's literature and art is equivalent to discussing the extent of the intellectual, cultural, and social human presence of these nations. Poetry is considered nothing but finds its most important model in Bedouin souls who live in the Arabian Peninsula. It is their widest and toughest outlet. Abu Firas Al-Hamdani says

Poetry is the record of Arabs

ever, and the title of lineage

With it, he belongs, and through it, he is known and revealed. Through the verses, the homes and their neighborhoods are recognized, and between its corridors are stories, feats, and achievements with eras of elevation and pride.

Emirati poetry came from the Arabic atmosphere that is mixed with land, history, and several eras. It connected the land and its history in formal poetry and colloquial poetry (or "folk poetry" as defined by critics), which was common within the Emirati people. This poetry is recognized because of the different aspects of the Emirati person between an eloquent tongue and a common dialect. The poet's thoughts were unconstrained. It was written with a smooth flow, like a stream in a rill in the middle of deserts and valleys. It expresses the thoughts of a person who lived and integrated with the land and its homes.

Emirates cultural and civilizational development is closely associated with the development in the Gulf and Arabian world because the Arabian Gulf represents a major access for the Arabian region into other cultures both Asian and international. This phenomenon has contributed to literary and poetic growth in the region in general and in the UAE in particular. Educational life has been developed with the increased activity of several educational institutions, along which we observed the progress of the human interest and how they contributed and interacted with their era's assistance. This influenced the progress in the modern Emirati literature and particularly in poetry.

The beginning of Emirates poetry was around the twenties of the last century. During this time, several pioneer poets shined, including Mubarak Al Aqili (1954), Salem Al Owais (1959), Ahmed Al Mannai (1990), Mubarak Al Nakhi (1982), Muhammad Nour Saif (1982), Ahmed Bin Salem (1986), Khalfan Bin Musbah (1946), and others whose names arrived without their poetry.

After the original poets, another group that came to be known as veterans. They followed these pioneers, met them, and lived their circumstances. Then, their time went beyond their predecessors. They became witnesses of the country's transition from phase to phase because of the new circumstances. These veterans include Saqr bin Sultan Al Qasimi (born in 1926), 
Sultan Bin Ali Al Owais (born in 1936), and Hamad Bu shehab (born in 1936). They paved the way for a classical subordinate traditional phase, with its distinctive artistic features and characteristics. It included the traditional column poetry with its unique artistic features with the mixing of traditional and modern culture in language, taste, and poetic sense, with a base in the values of Arabic poetry in its flourishing stages.

This classical stage was followed by a romantic phase, where the emotional restoration concepts risen from strict barriers. It transferred the soul closer to what it holds as it carried with it its emotional burdens. With that, numerous Emirati poets, as well as other poets in the Gulf and the Arabian world, have turned toward expressing this emotional instinct within. Among the leading romance poets was the Dr. Manei Saeed Al Otaiba, who sat on the throne of conscience, as well as other Emirati poets such as Sultan Al Owais and Hamad Khalifa Bu Shihab.

The period of Realism was associated with the reality of Arab and global society. It kept its pace with what was actually happening in the world and integrated poetry with its concerns and issues. Poets at that stage include Muhammad Sharif Al-Shaibani (1930-1998) and other poets during his time.

Modernization and experimental innovation came at a later stage. It gave the texts an aura of cohesion with modernist Arabic and the global experience. This was achieved by many Emirati poets, with renewal in the artistic template and suggestive artistic imagery. However, it remains attached to the emotional Romantic stage

Discussing a nation's literature and art is equivalent to discussing the extent of the intellectual, cultural, and social human presence of these nations. Poetry is considered nothing but finds its most important model in Bedouin souls who live in the Arabian Peninsula. It is their widest and toughest outlet. Abu Firas Al-Hamdani says

Poetry is the record of Arabs ever, and the title of lineage

With it, he belongs, and through it, he is known and revealed. Through the verses, the homes and their neighborhoods are recognized, and between its corridors are stories, feats, and achievements with eras of elevation and pride.

Emirati poetry came from the Arabic atmosphere that is mixed with land, history, and several eras. It connected the land and its history in formal poetry and colloquial poetry (or "folk poetry" as defined by critics), which was common within the Emirati people. This poetry is recognized because of the different aspects of the Emirati person between an eloquent tongue and a common dialect. The poet's thoughts were unconstrained. It was written with a smooth flow, like a stream in a rill in the middle of deserts and valleys. It expresses the thoughts of a person who lived and integrated with the land and its homes.

Emirates cultural and civilizational development is closely associated with the development in the Gulf and Arabian world because the Arabian Gulf represents a major access for the Arabian region into other cultures both Asian and international. This phenomenon has contributed to literary and poetic growth in the region in general and in the UAE in particular. Educational 
life has been developed with the increased activity of several educational institutions, along which we observed the progress of the human interest and how they contributed and interacted with their era's assistance. This influenced the progress in the modern Emirati literature and particularly in poetry.

The beginning of Emirates poetry was around the twenties of the last century. During this time, several pioneer poets shined, including Mubarak Al Aqili (1954), Salem Al Owais (1959), Ahmed Al Mannai (1990), Mubarak Al Nakhi (1982), Muhammad Nour Saif (1982), Ahmed Bin Salem (1986), Khalfan Bin Musbah (1946), and others whose names arrived without their poetry.

After the original poets, another group that came to be known as veterans. They followed these pioneers, met them, and lived their circumstances. Then, their time went beyond their predecessors. They became witnesses of the country's transition from phase to phase because of the new circumstances. These veterans include Saqr bin Sultan Al Qasimi (born in 1926), Sultan Bin Ali Al Owais (born in 1936), and Hamad Bu shehab (born in 1936). They paved the way for a classical subordinate traditional phase, with its distinctive artistic features and characteristics. It included the traditional column poetry with its unique artistic features with the mixing of traditional and modern culture in language, taste, and poetic sense, with a base in the values of Arabic poetry in its flourishing stages.

This classical stage was followed by a romantic phase, where the emotional restoration concepts risen from strict barriers. It transferred the soul closer to what it holds as it carried with it its emotional burdens. With that, numerous Emirati poets, as well as other poets in the Gulf and the Arabian world, have turned toward expressing this emotional instinct within. Among the leading romance poets was the Dr. Manei Saeed Al Otaiba, who sat on the throne of conscience, as well as other Emirati poets such as Sultan Al Owais and Hamad Khalifa Bu Shihab.

The period of Realism was associated with the reality of Arab and global society. It kept its pace with what was actually happening in the world and integrated poetry with its concerns and issues. Poets at that stage include Muhammad Sharif Al-Shaibani (1930-1998) and other poets during his time.

Modernization and experimental innovation came at a later stage. It gave the texts an aura of cohesion with modernist Arabic and the global experience. This was achieved by many Emirati poets, with renewal in the artistic template and suggestive artistic imagery. However, it remains attached to the emotional Romantic stage. 


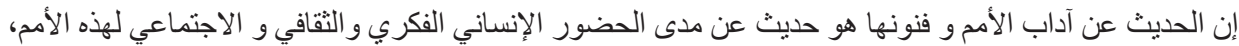

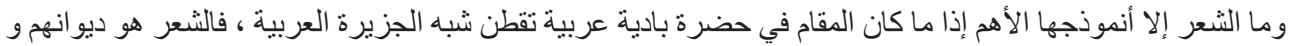

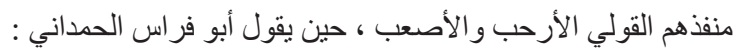

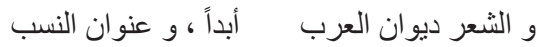

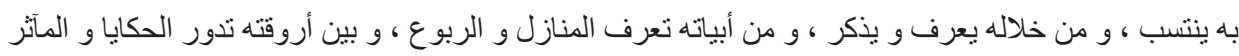
و المفاخر و عصور من الثمم و الإباء.

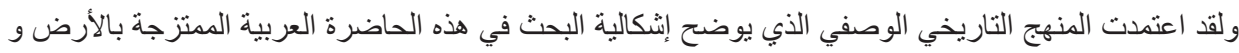

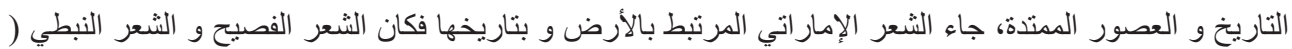

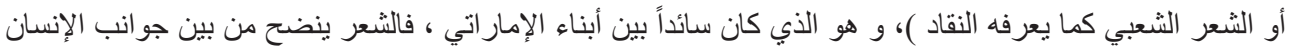

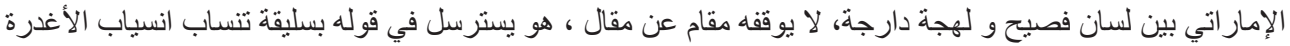

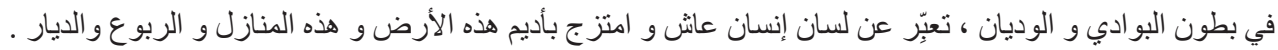

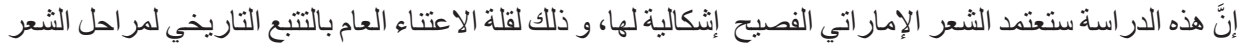

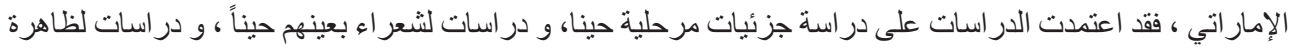

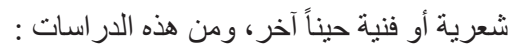

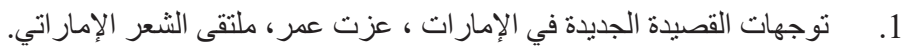

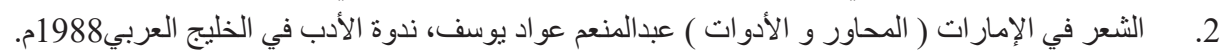

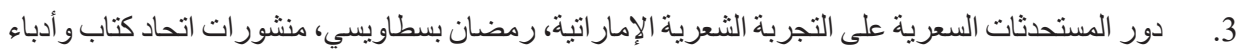

4. القصيدة الحديثة في الإمار ات، عبدالفتاح صبري ، دائرة الثقافة و الإعلام ،2005، 2007م.

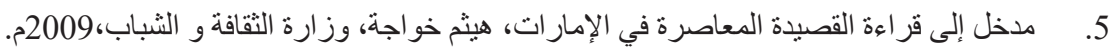

مما حفز البحث للسير وفق مسار التتبع التاريخي الوصفي للشعر الإمار اتي بشكل عام، و وضع تصور عام للمر احل

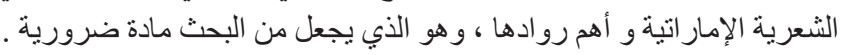

\section{أولا : الشعر الإمار اتي الفصيح وحضوره في شبه الجزيرة العربية}

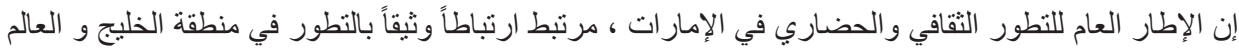

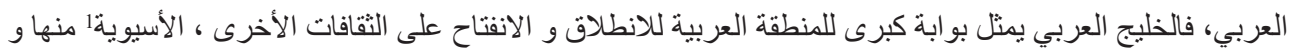

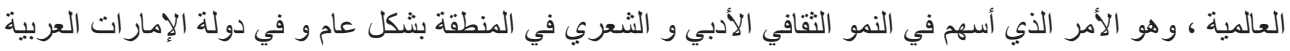

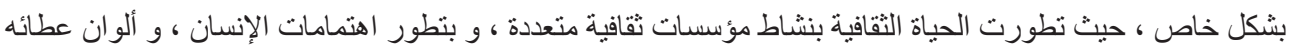

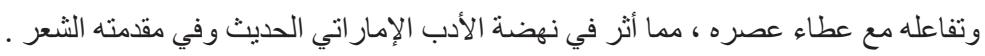

\section{ثانياً: ملمح تاريخي للشعر الإماراتي}

ومع انتشار الو عي الثقافي نتيجة عو امل عدة مثل : التو اصل الثقافي العالمي ، و تأسيس المؤسسات و الهيئات التي تعتني

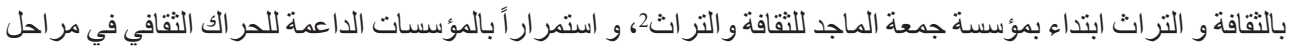

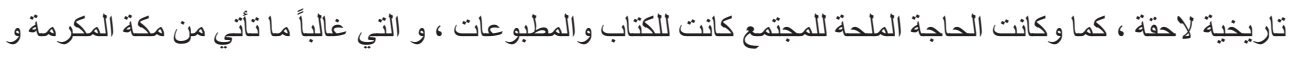

1 القد كان التو اصل من خلال الرحلات البحرية بين دبي وبومباي كبير الأثر في التواصل الثقافي بين الخليج و بين شبه القارة الهندية.

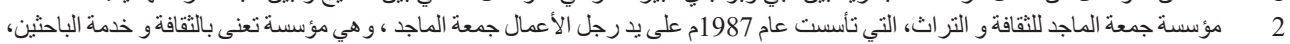

و تشجع الدراسات العلمية الرصينة. 
مصر عن طريق بومباي في الهن3، و لم تعرف المنطقة مؤسسات الطباعة ولا دور النشر إلا في فترة متأخرة ترقى إلى

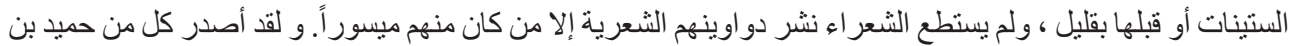

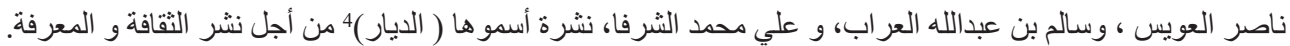

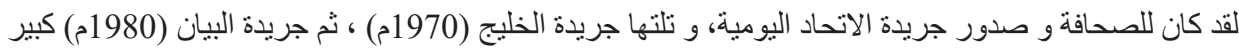

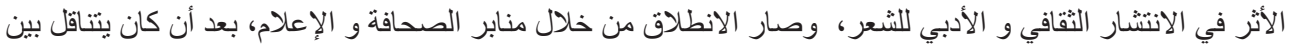

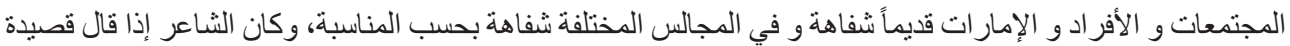

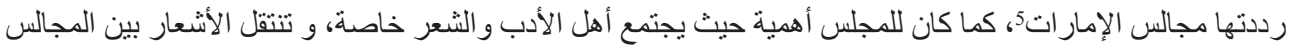

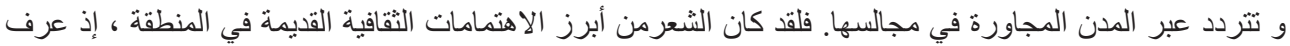

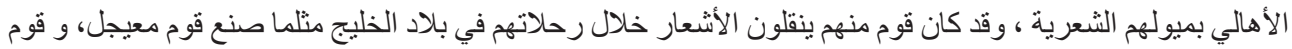

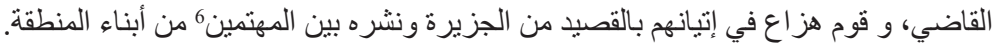

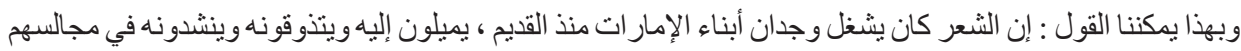

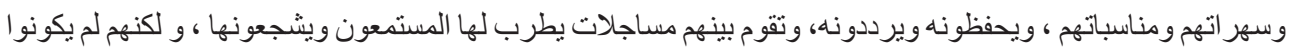
يدونونه في كتاب إلا قليلاً فضاع منه الكثير و النفيس .

\section{• البـدايـات}

إن بدايات الشعر في الإمار ات كانت في العشرينات7 من القرن الفائت ثقريباً ، حين برز عدد من الشعر اء الروَّاد ، و وهم

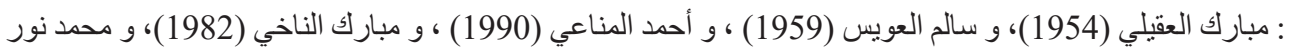

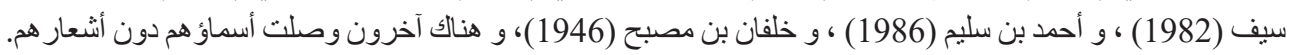
يمثل هؤ لاء الثعر اء طبقة واحدة متشابهة الميز ات، تلقت تعليماً واحداً هو التعليم القائم على العلوم الإسلامية و العربية،

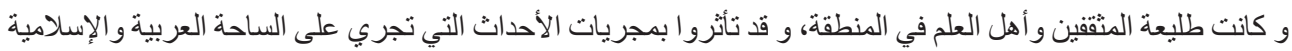

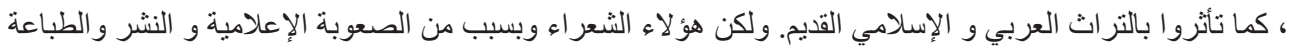

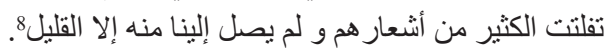

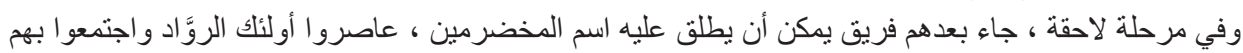

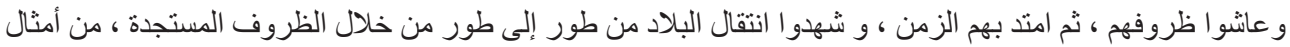

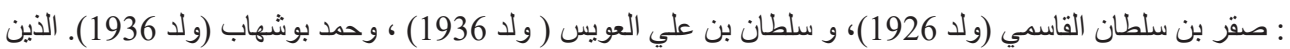

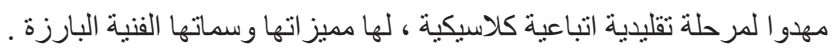

\section{1.المرحلة الاتباعية التقليدية ( الكلاسيكية )}

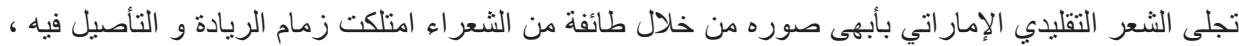

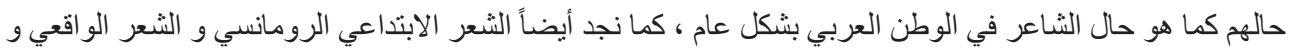

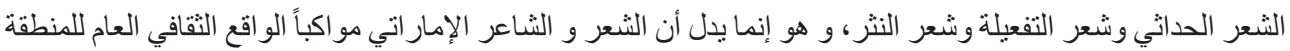

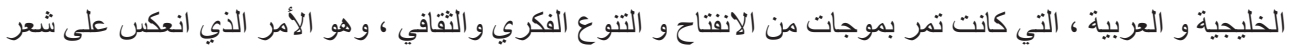

شاعر الإمار ات ، فأخصب فكرهو و قريحته وفق مرحلته ورؤيته.

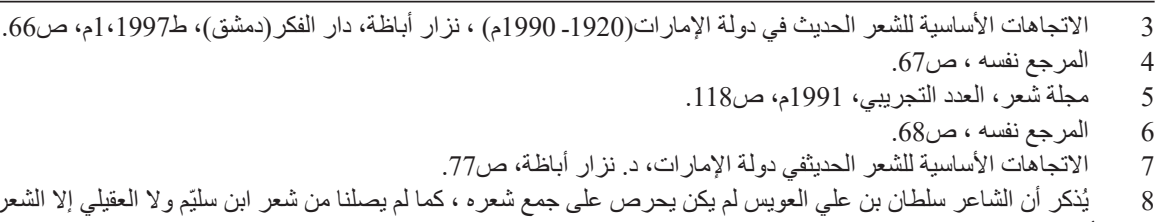

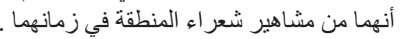




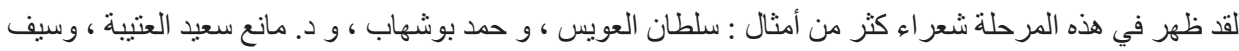

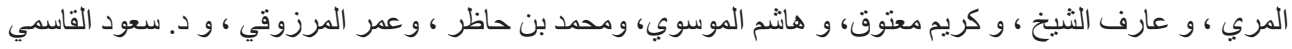

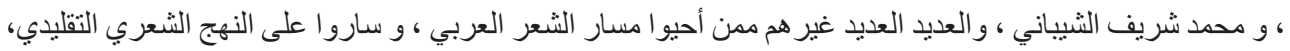

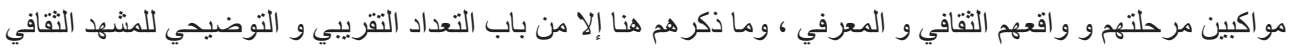

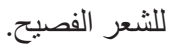

وتعد المدرسة الكلاسيكية هي مدرسة العمود الثعري التقليدي، و هي مرحلة أو مدرسة فنبة اتسمت بسمات عدة ، أهمها:

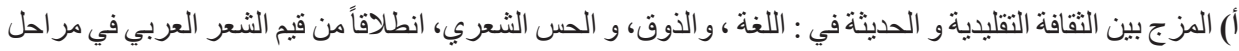

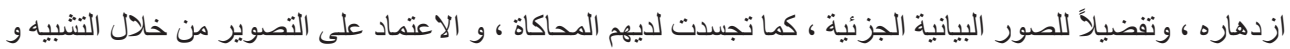

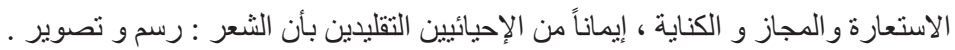

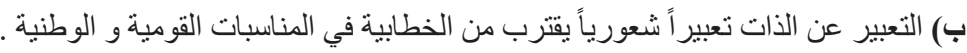

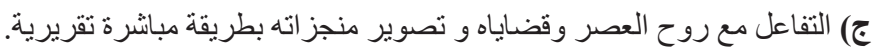

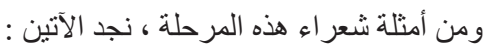

• الثـاعر راشد بن سالم السويدي( الخضر) الذي ولد في عجمان سنة 1905م، ونشأ بها وسافر إلى السعودية ، ثم عاد إلى وطنه بين الثارقة و و عجمان ، حتى الثى توفي بها أو اخر إكتوبر 1980، يقول فينه في قصيدنه:

$$
\begin{aligned}
& \text { رقت لحالي قلوب التركو العجم } \\
& \text { إلا الأحبة لا رقو او لا رحموا } \\
& \text { زادوا عتواً و حنَّت نحويَ النقم } \\
& \text { و كلما ازددت شوقا في محبتهم } \\
& \text { هم الثفاء لقلبي و السقام له } \\
& \text { أَنَّـــى لقلبـي أنْ يسلـــو ودادهم } \\
& \text { سل الرواسخ عن نومي و عن سهري } \\
& \text { لتعلم الحق إن أغرى بك الوهـــ } \\
& \text { لا بستوي النوم و الهجران و الألم } \\
& \text { نام الرفاق و قالو ا نم فقلت لهم } \\
& \text { قالو ا بلتك هموم الدهر قلت لهم } \\
& \text { قد يبتلي الهم من تسمو به الهمم } \\
& \text { جاء العو اذل من قومي بألسنة } \\
& \text { بيض حداد فأبدى ثلمهَا الصمح9 }
\end{aligned}
$$

$$
\text { • ماشم الموسوي }
$$

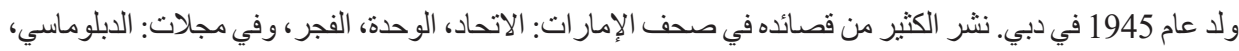

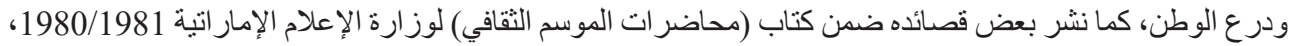
يقول في قصيدته ( في ظلال الشعر ): 


$$
\begin{aligned}
& \text { بروي القلوبَ هوى و الكون ألحانا } \\
& \text { تنهـدت عن عبير هـدهد البانسا } \\
& \text { من عبقر كان فيه الحسن ريانـا } \\
& \text { وتنجلي عن جمالل خف نشو انـا } \\
& \text { ضاف ييث الهوى نور اونير انـا } \\
& \text { باق على مسمع الأكوان أزمانا } \\
& \text { يد الأصيل من الإبداع ألوانا } \\
& \text { وجلوة الصبح تهدي الطير ألحانا } \\
& \text { يحنو على قلبها روحا وريحانا }
\end{aligned}
$$

شممت عطركِ إذ ينساب نديانا حييت روحك عبر السحر خافقة قدست حرفلك منسابا على وتر عر ائس تتو ارى في ندى خفر على ضفاف الرؤى من قلبها و هج وفي صلاة الندى من سحر ها أثر

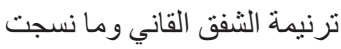

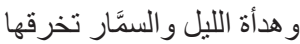
تهفو إلى عالم من فيضها عطر

فهو يسير في بنائه العمودي التقليدي ممتثلاً نهج الاتباعية التقليدية في التعبير عن القيم الثعرية و الفكرية الكلاسيكية المو ائمة لمرحلته . فير في

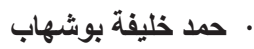

ولد في عام(1936م_ 1354ه) في إمارة عجمان ، اهتم في الشعر النبطي فكان أول من قدم برنامج شعري في برنامج

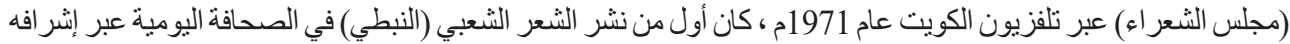

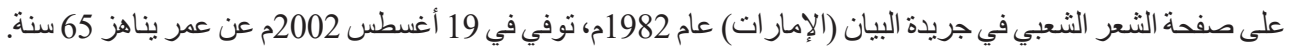

$$
\text { يقول في قصيدته معتز اً بالإسلام : }
$$

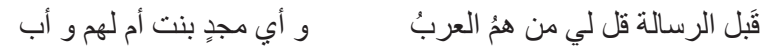

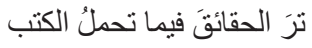
تعالَ فاستقرئ التاريخ أمثلة

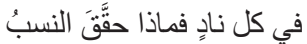
كان التفاخرُ بالأنساب ر ائدهم

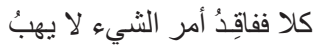
هل استطاعو ا به نوحيد أمتهم

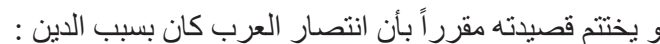

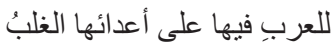

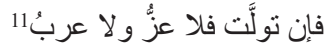

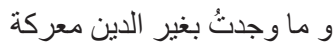

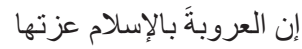

\section{• سلطان العويس بين الكلاسيكية و الروماتسية}

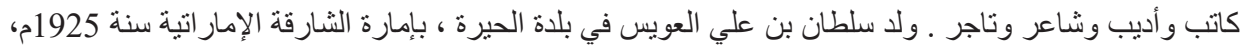

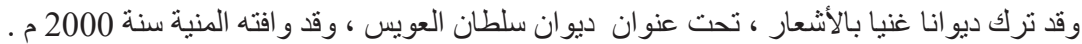

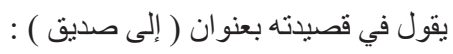

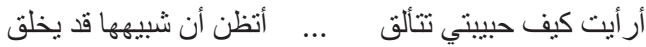

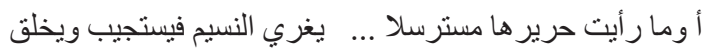

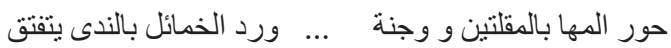

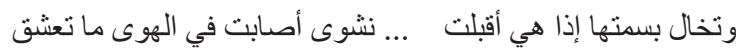

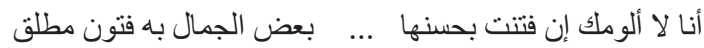




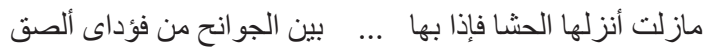

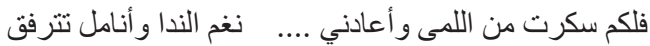

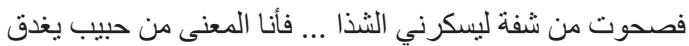

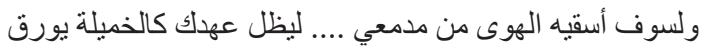
فرحي بها فرح السجين بلحظة ... عند اللقاء بأهلة إذ يطلق 12

\section{2.مرحلة التجديد الممزوجة بالوجدان ( الرومانسية )}

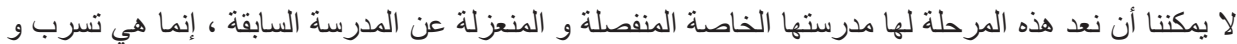

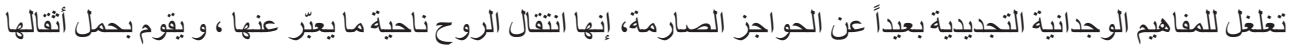

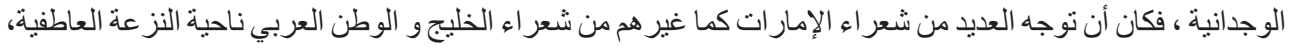

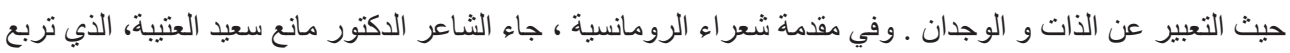

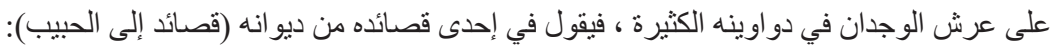

$$
\begin{aligned}
& \text { فاسمعيه يا فتـــــي } \\
& \text { هو ذا صوت فؤادي } \\
& \text { معلــناً في عبر اتي لـي في } \\
& \text { للنفوس الحائــــــرات13 } \\
& \text { و اشهدي صدق شعوري }
\end{aligned}
$$

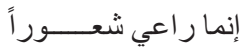

ويصل بشعوره و مشاعره وفيضهما إلى التطهر و التطهير :

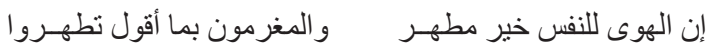

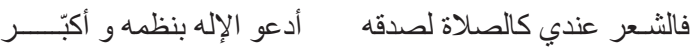

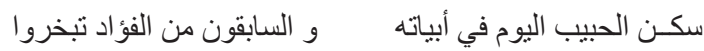

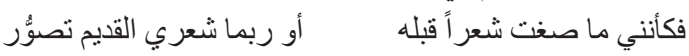

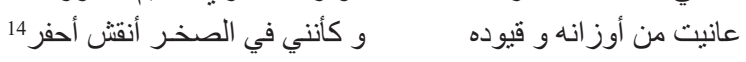

لقد تنقل الثعر اء بين مر احل فكرية متعددة ، وكل ذللك كان بدافع الحاجة الذاتية و النفسية ، فالشاعر الكلاسيكي الملتزم

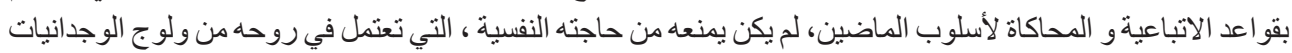

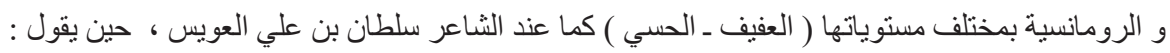

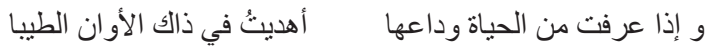

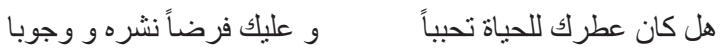

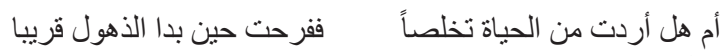

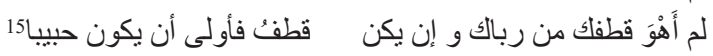

لقد كان للوجدانيات و الرومانسية حضور مستمر و إن تغير القالب الفني من العمود إلى شعر التفعيلة أو الثعر المرسل

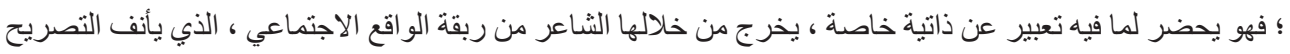

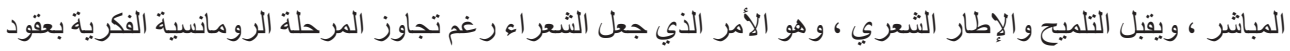

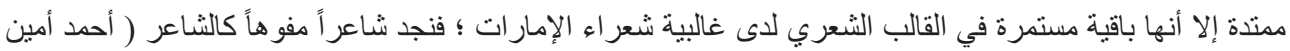




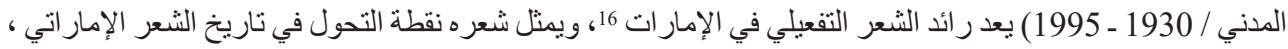

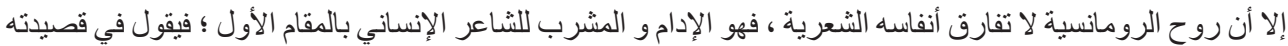

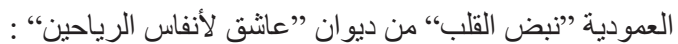

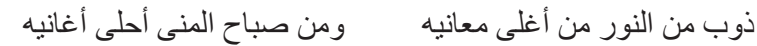

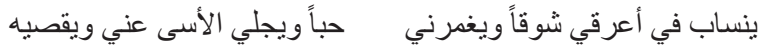

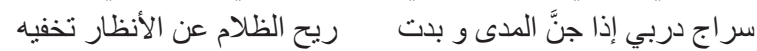

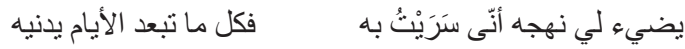

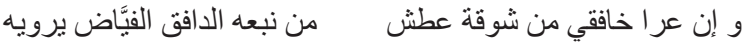

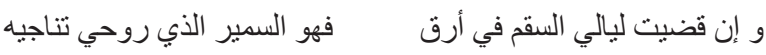

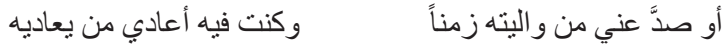
لم ألف غير الذي يفري به كبدي

وحين ينتقل إلى قصيدة التفعيلة ، فهو يوشحها بعنوان ” صباح دبوي“، نسبة إلى مدينة دبي التي يعيش فيها ، وفي فرجانها المفعمة الود الصباحي ، فيقول:

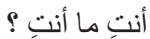

ألحنّ مبتكر

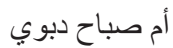
في حقولٍ من زهرج

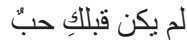

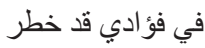
كنت حلماً

$$
\begin{aligned}
& \text { في ضمير الغيب عني مستتر } \\
& \text { فتجلّّى لي ابتساماً } \\
& \text { زاهر أ حين ظهر .ـ. }
\end{aligned}
$$

فهو يتغنى بدبي حباً وهياماً ، و يرى ربو عها حقو لاً من ز هر و ريحان .

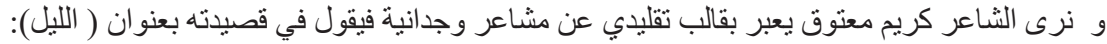
ما لي أقلب صورةًً

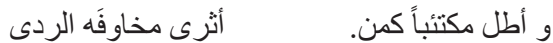

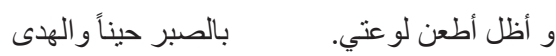

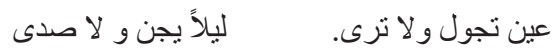

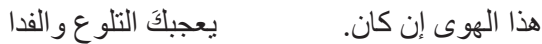

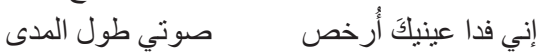

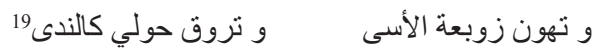


وفي مرحلة لاحقة ينتقل للتعبير برومانسية آسرة، وفق القالب التفعيلي، فيقول في قصيدته (مكاثشة ) :

$$
\begin{aligned}
& \text { خذي قِسطاً من النكر انِ } \\
& \text { بعضاً } \\
& \text { و ارجمي سهري } \\
& \text { و ردي عنف أخيلتي }
\end{aligned}
$$

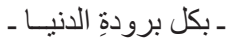

$$
\begin{aligned}
& \text { إذا جاءتلكِ فاعتذري } \\
& \text { دعي عنلكِ العتابَ المرَّ } \\
& \text { أدري أنني شبقُ القُّ }
\end{aligned}
$$

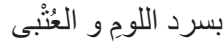

$$
\begin{aligned}
& \text { على الأحبابِ من صغري } 20
\end{aligned}
$$

\section{3. مرحلة الواقعية المرتبطة بالمجتمع و الواقع العربي والعالمي}

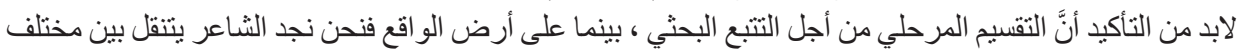

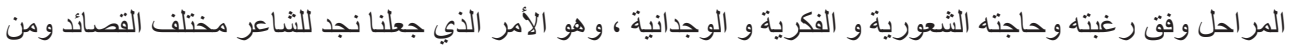

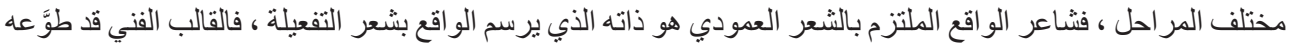

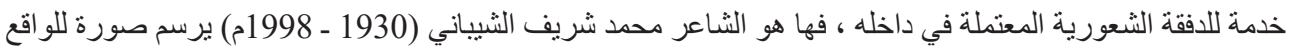
من خلال أبياته للتعبير عن القضية الفلسطينية :

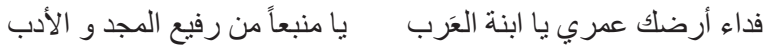

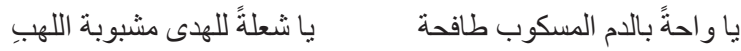

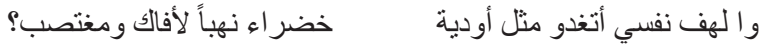

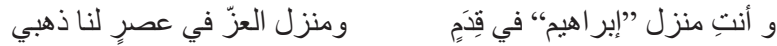

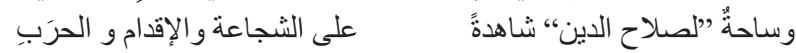

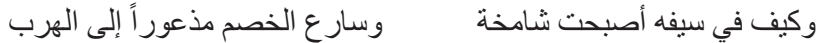

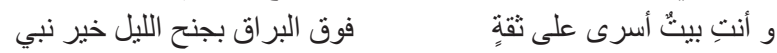

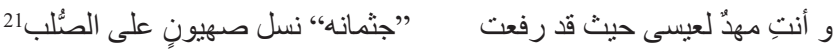

إنها الدققة الثعورية التي تتماهى مع الو اقع ، بينما القالب الشعري هو إطار لذلك المخزون.

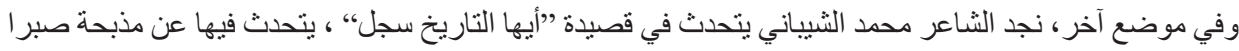
وشاتيلا، فيعبر عن الواقع الأليم :

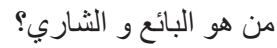
و من ر اهن في قبض الثمن

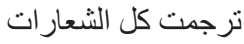

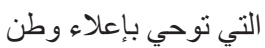
لعناء، لهباء، لغناء..

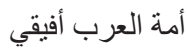




$$
\begin{aligned}
& \text { من سبات و هنات } \\
& \text { و احفظي من درس بيروت } \\
& \text { دروساً للحياة } \\
& \text { و استعيدي شرفاً } \\
& \text { غالته إسر ائيل عدو اناً و غفلة.. } \\
& \text { سوف تجتاح بلاداً } \\
& \text { زرعت فيها الثتات بنات } \\
& \text { و هي تسعى باطر اد } \\
& \text { ترسم النيل حدوداً } \\
& \text { لينابيع الفرات222 }
\end{aligned}
$$

إنَّ الثاعر الإمار اتي، اندمج مع و اقعه الوطني كما اندمج مع قضايا الأمة ، فنرى الثاعر هاثم الموسوي ، يسطِّر

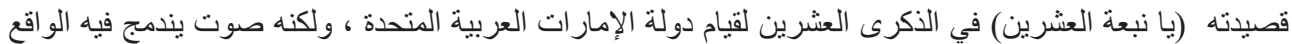

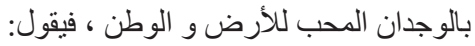

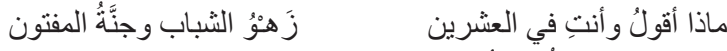

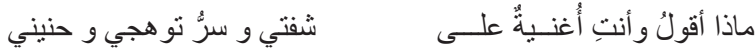

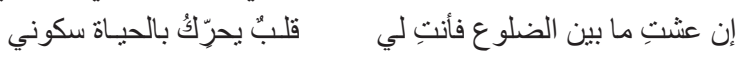

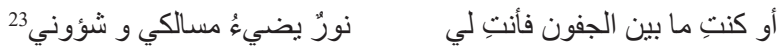

إن شعر اء الو اقع كانو ا مندمجين مع و اقعهم العربي و الإسلامي ، لم ينفكو ا عنه ، إنهم ينتمون إليه بل يتنفسونه ويحلمون

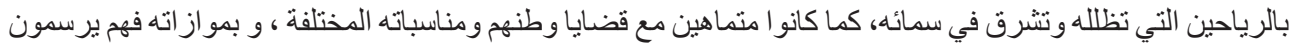
و اقعهم بخيوط وجدانية رومانسية شفيفة.

\section{4. مرحلة التجايد و الثعر التجريبي مندي}

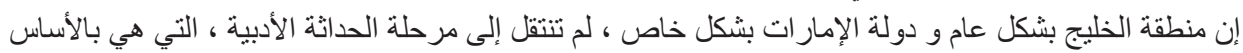

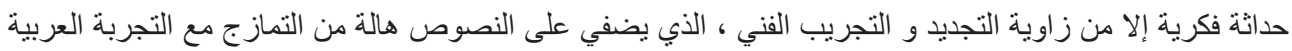

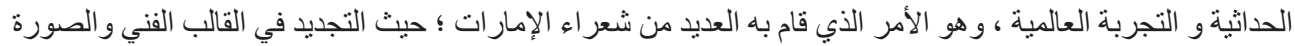

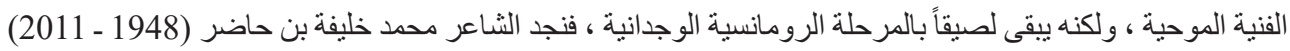

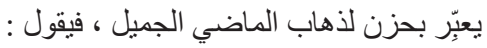

$$
\begin{aligned}
& \text { يا صاحبي ماذا تريد } \\
& \text { نضبت بنابيع المنى مادي } \\
& \text { و هوت على الأرض الخز امى لئ لئن } \\
& \text { ويل لمن غدروا بها التها } \\
& \text { ماذا جنت } \\
& \text { حتى تجازى بالزو ال } \\
& \text { وتنتهي بعد الحياة إلى الدحال24 }
\end{aligned}
$$


إنه يرسم ملامح الحزن المنتشر في أروقة قلبه على الزمن الجميل ، و يعتمد لغة التجريب الفني من حيث الصور الموحية، ولكن تبقى اللغة الو اضحة سيدة القصيدة.

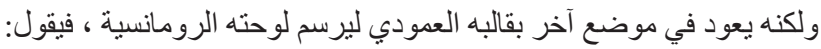

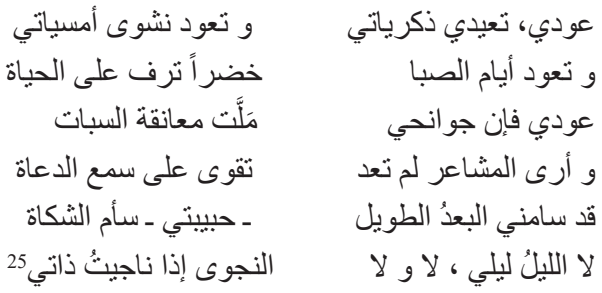

بينما نجد الثاعر حبيب الصايغ في قصيدته "نوفمبر ـ أكتوبر“، التي يوحي عنو انها بعودة الزمان إلى الوراء، أو محاولة استبقاء الحياة ، فيقول : إنَّ الخر افة قد شرشت في دمي و اصطفتني و وها تستنفرُ الخيل في جسدي و تداعت عمري حتى يجيى

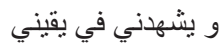
لسوف ير الك تمرين بين المفاصل و القلب صاخبة و تنامين بين النو اصل و الرمح نوماً جموحاً ألا إنها سنة الحلم26

إن تجربة الثاعر حبيب الصايغ من المكن أن نقول عنها تجربة حداثية أدبية ، تتجاوز الصور الاعتيادية إلى صور

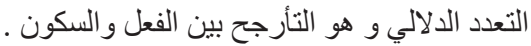
كما نجد التجديد و الانتقال الفني لدى الثاعر شهاب الغانم في قصيدتها الأمواج)، حين يقول:

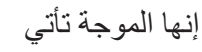
إنها الموجة تذهب الموجه يا حبيبي هكذا الدنيا صروف تنتقلب إن قسا الدهر علينا

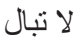

لا تقابله حزيناً

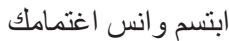

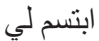
آه ما أحلى ابتسامك لئي 
و نجد الثاعر إبر اهيم الهاشمي في قصيدته ( الغمغمة)، يوظف القالب الفني الجديد للتعبير عن و اقع التو اصل ، فيقول :

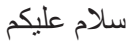

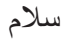
سلام أيها المثنخون بجرم التوم التومل سلام عليكم سلام سلام وقلبي هيام خيمة على ضفتين نهز من الحزن جرح.. و عين قلب يعدده النصل

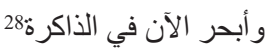

و أما الأنثى المبحرة في التجريب ، و الباحثة عن الانعتاق و الرغبة في التجديد و الإفادة من الحداثة و المغايرة، فنجد

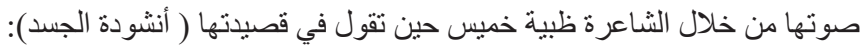
فحيح ... وشهقة و همهمات تمو ء، تضيع ويندفع الأقحوان وينسل الفيروزُ من العاج.. كما يفعل اللؤلؤ أي وشاح يغطي العظام

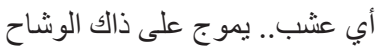

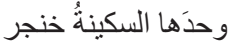
وحده القلب صحر اء.

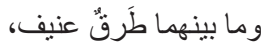
دبيب، ودقات القبائل عند المغيب 29

بينما تنساق في أحلامها الوردية ، و و وعود الرؤى الثفيفة الثاعرة ميسون صقر في قصيدنها (يبادرني الحلم) فتقول:

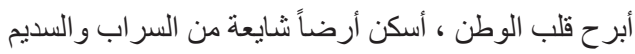
أنا المشحونة بالعندليب وصديح الغناء تكورني صحبتي دائرة من الفقاع، فأكون ذابلة

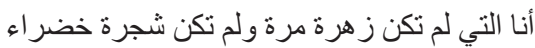
إنما اللون المز هر يفرش قبلتي و الإخضر ار لا يعرف كيف يستقيم 30 


$$
\begin{aligned}
& \text { و نجد الثاعرة نجوم الغانم في قصيدتها ( استقبلت وقعاً ليس بغريب) تقول: } \\
& \text { استقبلت جمو عاً و أساطير } \\
& \text { تسير بمحاذاة ليل } \\
& \text { ماذا لو أُقِّبّل هذا الغبار } \\
& \text { و أكون أختاً }
\end{aligned}
$$

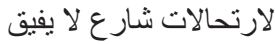

$$
\begin{aligned}
& \text { إلا بجانب رأسي } \\
& \text { لكني سأتأبط ذر اع شيخ } \\
& \text { لم بعد يعرفني } \\
& \text { و أدعوه لتناول عثاء في مقبرة31 }
\end{aligned}
$$

و تأتي الثاعرة الهنوف محمد لترسم صحر اءها القاحلة في قصيدتها ( الصحر اء تشبهني) ، حيث الاغتر اب و التيه، فتقول:

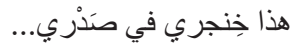

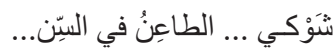
فاقتَلِعـوه ...

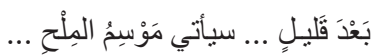

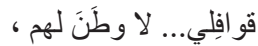

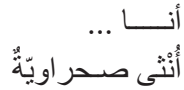

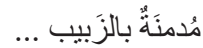

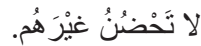

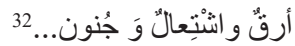

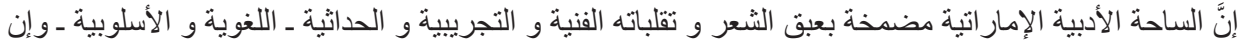

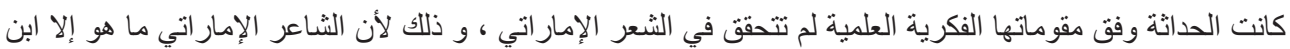
بيئته، فهو يتماهى مع قضاياه وو اقعه و كينونته الاجتماعية.

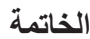

إن الأدب الإمار اتي بشكل عام ، و الشعر الإمار اتي بشكل خاص كان وماز ال حاضر اً في وجدان و و اقع الإنسان

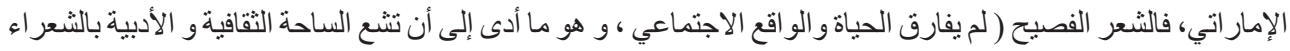

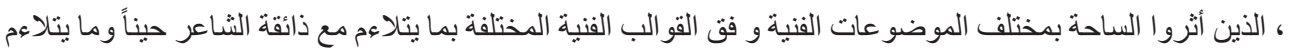

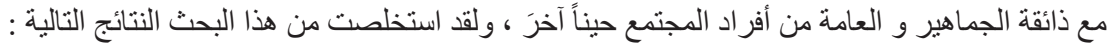

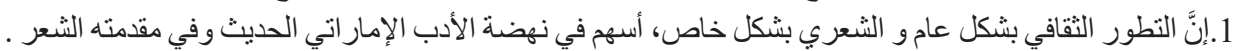

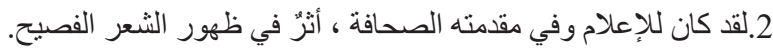
3.إن الشعر كان يشغل وجدان أبناء الإمار ات منذ القديج. 4.إنّ بدايات الشعر في الإمار ات كانت في بدايات القرن العشرين ، حين برز عدد من الشعر اء ، مثل: مبارك العقيلي 


$$
\text { ، و وسالم العويس، و أحمد المناعي ، و مبارك الناخي و غير هم . }
$$

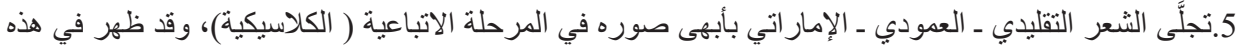

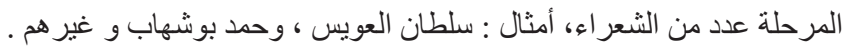

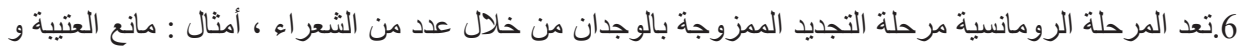

$$
\text { أحمد المدني و غير هم . }
$$

7. لقد برزت مرحلة الو اقعية مرتبطة بالمجتمع و الو اقع العربي و الإسلامي ، ومن شعر اء هذه المرحلة : محمد

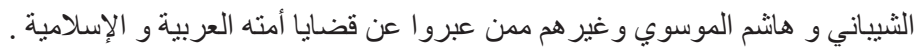

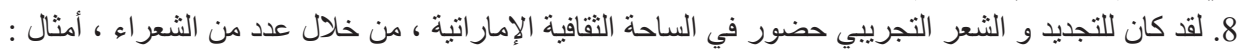

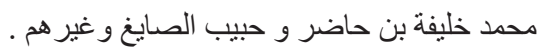

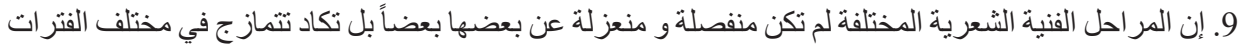
التاريخية ، ولكن البحث اتخذ التقيم للمر احل من أجل التوضيح و إعطاء تصوّر عن شعر و شعر اء كل مرحلة .

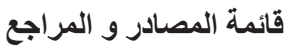

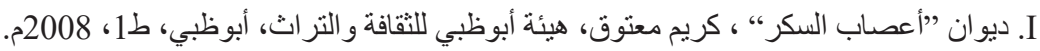
II

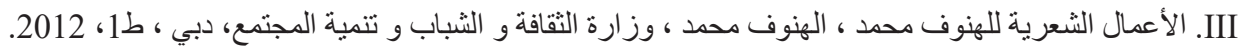

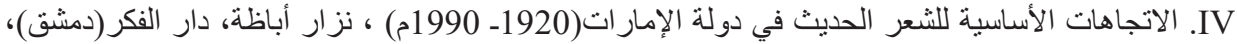

V شعر اءء دولة الإمار ات العربية المتحدة( در اسة و ببلو غر افيا)، د. يوسف نوفل، ندوة الثقافة و العلوم ، دبي ، ط1،

http://www.albabtainprize.org/encyclopedia/poet/1874.htm ، مؤسسة جائزة عبد العزيز البابطين. VI

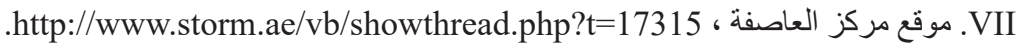
طا2 سلسلة أعلام من الإمار ات 18 (شعر اء من الإمارات) ، د. شهاب الغانم، مؤسسة سلطان العويس الثقافية ، دبي ، ط2015، 2015. ط. قصائد من الإمار ات، منشور ات اتحاد كتاب و أدباء الإمار ات العربية المتحدة، ط1، 1986م.

Peer-review: Externally peer-reviewed.

Conflict of Interest: The author has no conflict of interest to declare.

Grant Support: The author declared that this study has received no financial support.

\section{References}

Abdulaziz Saud Al-Babtain Cultural Foundation, http://www.albabtainprize.org/encyclopedia/poet/1874.htm. el-Henûf Muhammed, A 'mâlu'ş-şi 'riyye li'l-Henûf Muhammed, Dubai: Vizâretu's-sekâfe ve'ş-şebâb ve tenmiyeti'l-muctema', 1.bs., 2012.

Hâşim b. el-Huseyn el-Mûsevî, Dîvânu 'Enînu'l-Kavâfî', Dımaşk: Dâru'l-fetâtli't-tıbâ'a ve'n-neşr ve't-tevzî, 1.bs., 2004.

Kerîm Ma'tûk, Dîvânu 'A 'sâbu's-sukker', Dubai: Hey’et Ebu Dabî li’s-sekâfe ve’t-turâs, 1.bs., 2008.

Komisyon, Kasâid mine 'l-İmârât, Dubai: Menşûrâtu İttihâdi Kuttâb ve Udebâi'l-İmârâti'l-arabiyye el-Muttehide Mevk1'u merkezi'l-‘âsife, http://www.storm.ae/vb/showthread.php?t=17315. 
Nizâr Abaza, el-İtticâhâtu'l-esâsiyye li 'ş-şi 'ri'l-hadîs fì devleti'l-İmârât (1920-1990), Dımaşk: Dâru'l-fikr, 1.bs, 1997.

Şihâb el-Ğânim, Silsiletu a 'lâm mine'l-İmârât (Şu 'arâ mine'l-İmârât), Dubai: Muessese Sultân el-Uveys es-Sekâfiyye, 2.bs., 2015.

Yûsuf Nevfel, Şu 'arâu devleti'l-İmârâti'l-Arabiyye el-Muttehide (Dirâse ve Bibliyografya), Dubai: Nedvetu'ssekâfe ve'l-ulûm, 1.bs., 1994. 
